\title{
Intervención de enfermeria en el autocuidado de pacientes diabeticos tipo II durante el primer nivel de salud
}

\section{Intervention of nurse in the autocare of diabetic patients type II during the first level of health}

\author{
Dolores Mirella Cedeño-Holguin \\ doloresmirella@hotmail.com \\ Universidad Estatal del Sur de Manabí, Jipijapa \\ Ecuador \\ https://orcid.org/0000-0002-8572-0327 \\ Franklin Antonio Vite-Solorzano \\ antuanvit@hotmail.com \\ Universidad Técnica de Manabí, Portoviejo \\ Ecuador \\ https://orcid.org/0000-0002-6732-7994 \\ Aida Monserrate Macias-Alvia \\ aidita.macias@hotmail.com \\ Universidad Estatal del Sur de Manabí, Jipijapa \\ Ecuador \\ https://orcid.org/0000-0001-5290-4317 \\ Bolívar Cevallos-Jácome \\ boly1404@gmail.com \\ Universidad Técnica de Manabí, Portoviejo \\ Ecuador \\ https://orcid.org/0000-0002-6334-1385
}

Recibido: 09 de noviembre de 2019

Aprobado: 15 de diciembre de 2019

\section{RESUMEN}

El presente artículo tuvo como objetivo analizar la intervención de enfermería en el autocuidado de pacientes diabéticos tipo II durante el primer nivel de salud. La metodologia fue de tipo documental y descriptivo. La población la constituyeron 45 pacientes diabéticos que pertenecen al club de diabéticos del Centro de Salud "Daniel Acosta Rosales" tipo "B". Para la obtención de la información se aplicaron encuestas y entrevistas. Los resultados permitieron concluir que la enfermería posee un rol determinante en el autocuidado de los pacientes diabéticos, al permitir prevenir, retrasar o disminuir la probabilidad de desarrollar complicaciones que generan tanto 
impacto en el ámbito social, psicológico y económico de las personas afectadas como a su entorno familiar.

Descriptores: Enfermería; Diabetes tipo II; Autocuidado y pacientes.

\begin{abstract}
This article aimed to analyze the intervention of nursing in the self-care of type II diabetic patients during the first level of health. The methodology was documentary and descriptive. The population consisted of 45 diabetic patients belonging to the diabetic club of the "Daniel Acosta Rosales" type "B" Health Center. Surveys and interviews were applied to obtain the information. The results led to the conclusion that the infirmary has a decisive role in the self-care of diabetic patients, by allowing to prevent, delay or decrease the likelihood of developing complications that have so much impact on the social sphere, of those affected as well as their family environment.
\end{abstract}

Descriptors: Nursing; Type II diabetes; Self-care and patients.

\title{
INTRODUCCIÓN
}

La Diabetes Mellitus 2 (DM2) es una enfermedad crónico-degenerativa que representa un grave problema de salud pública y de alto costo que afecta a aproximadamente 250 millones de personas en el mundo cuyas complicaciones agudas y crónicas constituyen uno de los retos más importantes a los que deben hacer frente los sistemas de salud. De la misma forma que el resto de las enfermedades crónicas está vinculada a factores conductuales, nutricionales y de influencia medioambiental, que en interacción con condicionantes genéticas causan la entidad. El modo y estilo de vida occidental, con disminución de la actividad física y la inadecuada alimentación por consumo excesivo de alimentos de elevado contenido calórico, están entre las causas principales de esta epidemia. (Casanova y col, 2017)

La prevalencia de DM2 ha alcanzado proporciones epidémicas en todo el mundo; de acuerdo con la Federación Internacional de Diabetes (FID), hasta el 2017 existían 425 millones de personas con esta enfermedad, de los cuales el 79\% vivía en países de ingresos bajos y medios (Hernández y col, 2013). La DM2 es responsable de casi el $50 \%$ de las amputaciones no traumáticas de miembros inferiores, una importante 
proporción de los infartos agudos de miocardio, ataques cerebrovasculares e insuficiencia renal crónica en tratamiento dialítico, constituyendo la primera causa de ceguera no traumática en adultos. (Ministerio de Salud de Argentina, 2015)

Las opciones para el control de la DM se mueven en las cuatro direcciones del quehacer de la medicina: promoción, prevención, curación y rehabilitación. Una herramienta básica para el control de la DM es la educación para la salud, proceso que se da durante toda la vida, que enseña al individuo a conocer mejor su cuerpo y a cómo mantenerlo. De manera que con un panorama donde la prevalencia de la DM aumenta en todos los grupos de edad, la promoción de la salud y la educación para la salud son los ejes para el paso del concepto negativo de la salud a una visión positiva donde la base del conocimiento y de las destrezas habilitan al individuo y a la comunidad para hacer elecciones responsables en cuanto a sus prácticas cotidianas (Casanova y col, 2017).

El déficit de autocuidado en pacientes diabéticos refleja la necesidad de profundizar en el nivel de conocimientos hacia prácticas para el autocontrol de la enfermedad, a partir del sistema de apoyo educativo, como parte central de las responsabilidades del profesional de enfermería (Ulloa y col, 2017). Su prevalencia continúa en ascenso desmesurado en todo el mundo como resultado de la intervención de una serie de factores socioculturales, entre los que destacan: el progresivo incremento de la obesidad, el sedentarismo y la carencia de conocimientos sobre su autocuidado favorecen el aumento de complicaciones multisistémicas en los pacientes diabéticos y se convierten éstas en las motivaciones suficientes para realizar la intervención. Ante este panorama, en el siguiente artículo se analizó la intervención de enfermería en el autocuidado de pacientes diabéticos tipo II durante el primer nivel de salud.

\section{DESARROLLO}

La diabetes es una enfermedad complicada que requiere de una vigilancia y unos cuidados constantes pero que, si se tiene la información necesaria y se siguen ciertos hábitos saludables, no incide apenas en la calidad de vida de aquellas personas que la sufren. 


\section{Clasificación de la diabetes}

El Comité de Expertos de la Asociación Americana de Diabetes (ADA, 2005) y de la Organización Mundial de la Salud (OMS, 2011), han propuesto una nueva clasificación que contempla 4 grupos:

Diabetes mellitus tipo 1. También denominada diabetes insulinodependiente, es ocasionada por la destrucción de la célula $B$ de los islotes de Langerhands pancreáticos, provocado por alteraciones inmunológicas o de causa desconocida (idiopática). Se caracteriza por su insulinodependencia, constituye el $10 \%$ de las DM primarias y suele desarrollarse antes de los 30 años. Tiene un inicio clínico agudo o subagudo con tendencia a la cetosis que puede derivar en cetoacidosis. (Girone, 2005).

Diabetes mellitus tipo 2. Son aquellas formas con resistencia insulínica predominante y, eventualmente, una deficiencia relativa de secreción de insulina, de etiología esencialmente desconocida. La causa es, por tanto, una combinación de resistencia a la acción de la insulina (generalmente asociada a obesidad) y una inadecuada respuesta secretora de insulina compensatoria. Intervienen en su aparición factores genéticos y ambientales. Presenta un factor hereditario muy importante y suele desarrollarse después de los 40 años en personas obesas. No existe tendencia a la cetosis y al menos en su inicio, no suele ser dependiente de la insulina. La denominación de Diabetes mellitus no insulinodependiente para hacer referencia a la DM tipo 2, no se recomienda ahora puesto que muchos de estos pacientes acaban precisando la administración de insulina (Galvis, 2007).

Otros tipos específicos de diabetes. Llamado "Otros tipos específicos de diabetes" donde se incluyeron los casos cuyo defecto básico es conocido y puede ser identificado. En este se incluyen tipos de diabetes que tienen déficit de insulina por destrucción de las células $B$, aunque no de causa autoinmune, o casos de diabetes tipo 2 por resistencia a la insulina, por defectos genéticos conocidos. (Escobar, 2000). 
Diabetes mellitus gestacional. La definen simplemente por el hecho de aparecer durante el embarazo; A partir de la segunda mitad del embarazo, se produce una mayor secreción de hormonas con acción diabetógena (lactógeno placentario, estrógenos, progesterona) que aumentan la tendencia a la hiperglucemia, provocando la aparición de diabetes gestacional entre el 2 y el $4 \%$ de las gestantes, inicialmente no diabéticas. Es recomendable la realización de un test de tamiz (Test de O'Sullivan) en gestantes con riesgo moderado y alto (edad superior a 35 años, antecedentes de diabetes gestacional, obesidad, glucosuria, antecedentes familiares de diabetes en primer grado) a las 24-28 semanas de embarazo.( ALAD, 2011) También, cabe destacar que Baca y col (2008), explica que es una enfermedad poligénica compleja en la que las variantes genéticas comunes interactúan con factores ambientales para desenmascarar la enfermedad, el mismo autor indica que la vida de una persona diabética debe girar sobre cuatro pilares básicos:

a.-Educación, formación e información: La diabetes es la única enfermedad crónica que, aunque se conozcan sus causas y tratamiento, sigue creciendo, por lo que formar e informar a los pacientes, es vital para que los diabéticos puedan conocer su patología y sepan qué deben hacer, por lo que existe la necesidad de que los especialistas tienen que indicar a cada paciente el criterio que debe seguir, por lo que hacerse revisiones de forma periódica y acudir habitualmente al especialista es también un hábito que debe ser incorporado para evitar complicaciones innecesarias y llevar una vida normal.

b.- Alimentación: una buena alimentación, junto con el ejercicio, debe formar parte, como conjunto, de la forma de vida de las personas diabéticas. A pesar de la limitación de algunos alimentos, algunos especialistas coinciden en opinar que un diabético no debe privarse de nada, es decir, no debe eliminar de su dieta ningún alimento, lo que sí debe hacer es ingerir con moderación aquellos productos que puedan elevar los niveles de glucosa en sangre y las grasas, como los dulces, pero eso no quiere decir que se conviertan en alimentos totalmente prohibidos. 
c.- Ejercicio: hacer ejercicio y llevar una buena alimentación son los ejes principales de una vida saludable, por lo que en personas con diabetes cobra mucha importancia. Es importante conocer que no existen ejercicios específicos para personas con diabetes, sino que cada uno debe practicar deporte en la medida en que pueda, es decir, hay que tener en cuenta factores como la edad, la condición física y otros problemas o enfermedades. La Organización Mundial de la Salud recomienda a las personas diabéticas realizar 30 minutos de ejercicio físico al día, aunque sea de intensidad leve.

d.- Medicamentos: seguir el tratamiento de forma correcta, sin interrupciones, es muy importante para evitar complicaciones. Los fármacos, junto con el resto de hábitos, reducen drásticamente los riesgos que conlleva la enfermedad y permiten a los pacientes llevar una vida normal.

\section{MATERIALES Y MÉTODOS}

La metodologia fue de tipo documental y descriptivo. Para Hernández y col (2014), la investigación descriptiva refiere a aquellos estudios que describen a las variables en estudios tal como se presentan en su estado natural. Por su parte, García (2000), señala a la investigación documental, como un proceso estratégico en donde el investigador busca a través de la consulta en distintas fuentes documentales, recabar los datos e información teórica existente sobre el tema que pretende estudiar, a fin de obtener material intelectual y científico en donde pueda descansar el desarrollo de la investigación científica que desea realizar. Para la obtención de la información a describir, se aplicaron encuestas y entrevistas, relacionadas con la determinación de las intervenciones de enfermería aplicadas a los pacientes que pertenecen al club de diabéticos del Centro de Salud "Daniel Acosta Rosales" tipo "B" y la relación de las características sociodemográficas con sus complicaciones. La muestra estuvo conformada por 45 pacientes diabéticos.

\section{RESULTADOS Y DISCUSIÓN}

A continuación, se presenta el análisis y discusión de los resultados de los datos obtenidos relacionados con la frecuencia de intervención de los profesionales de 
enfermería en el autocuidado de los pacientes diabéticos. Posterior a la aplicación de los instrumentos a los pacientes investigados, los mismos se presentan en tablas con sus respectivos gráficos.

Tabla 1. Análisis frecuencial del sexo en pacientes diabéticos tipo II.

\begin{tabular}{lcc}
\hline $\begin{array}{c}\text { Alternativa/ } \\
\text { Análisis }\end{array}$ & Frecuencia & Porcentaje \\
\hline F & 30 & 67 \\
M & 15 & 33 \\
Total & 45 & 100,0 \\
\hline \hline
\end{tabular}

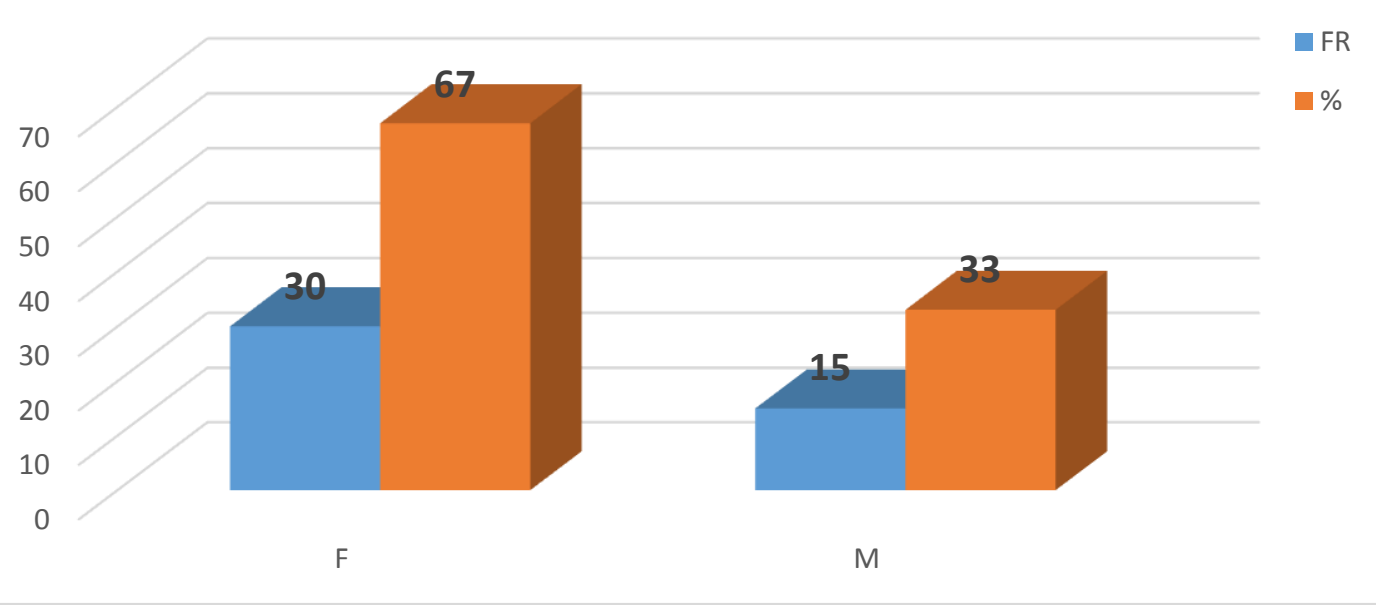

Grafico 1. Análisis frecuencial del sexo en pacientes diabéticos tipo II.

En la tabla y grafico 1, se presenta el análisis frecuencial del indicador sexo de los pacientes diabéticos tipo II que asistieron al centro de salud Daniel Acosta. En la misma se indica que el $67 \%$ se corresponden al sexo femenino. Mientras que resto, representado con el 33\% eran del sexo masculino. Cabe destacar que un alto porcentaje de pacientes eran con edades comprendidas entre 60-65 años de edad y el $82 \%$ de los participantes presentaban patologías asociadas, entre las que sobresale la hipertensión arterial con el 47\%. 
Tabla 2. Análisis frecuencial de la procedencia en los pacientes diabéticos tipo II.

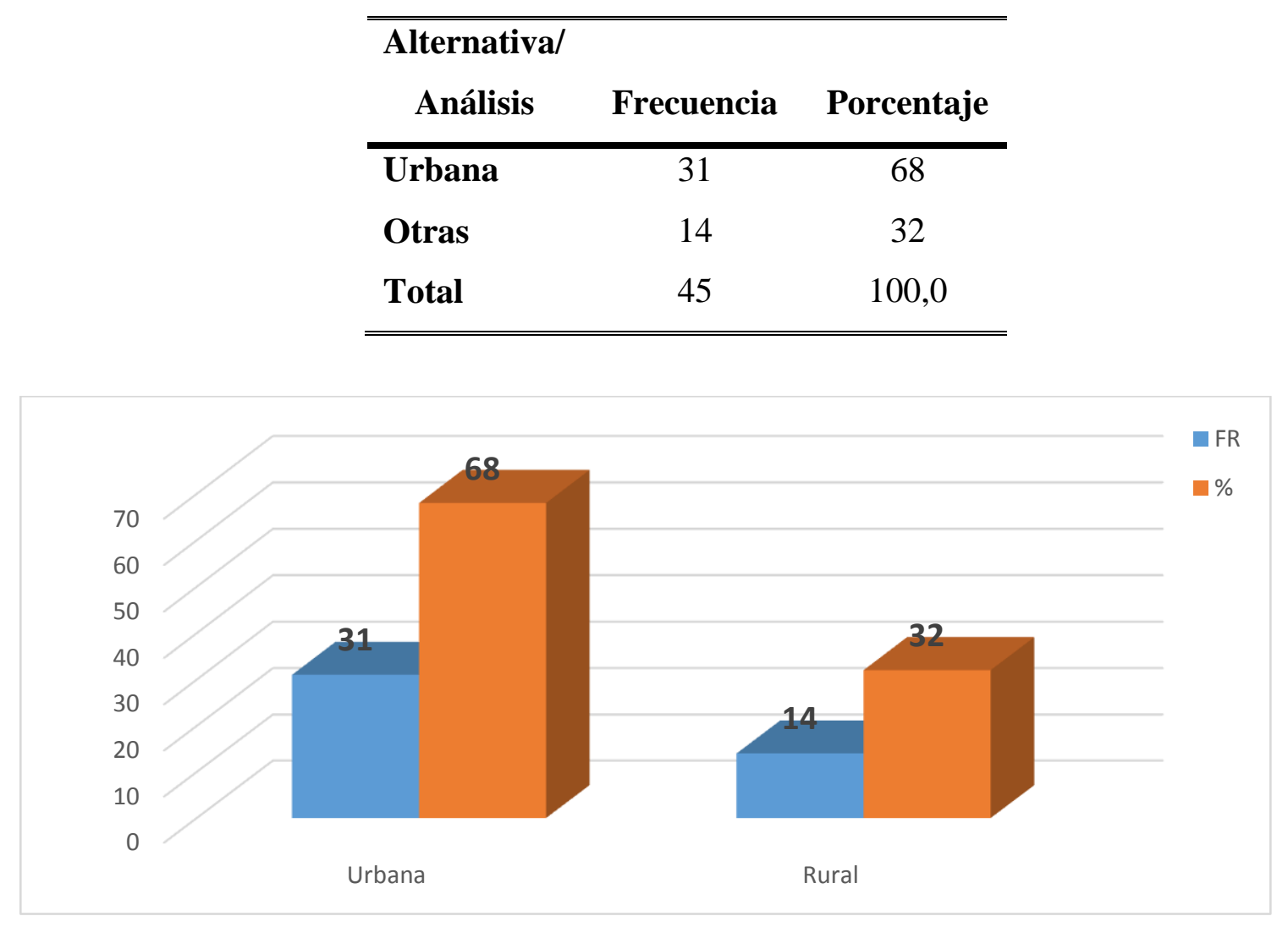

Grafico 2. Análisis frecuencial de la procedencia en los pacientes diabéticos tipo II.

En la tabla y grafico 2, se presenta el análisis frecuencial del indicador procedencia de los pacientes diabéticos tipo II que asistieron al centro de salud Daniel Acosta. En la misma se indica que el $68 \%$ son de procedencia urbana y el $32 \%$ eran de otras procedencias. 
Tabla 3. Intervención de enfermería en la alimentación de pacientes diabéticos tipo II.

\begin{tabular}{lcc}
\hline $\begin{array}{c}\text { Alternativa/ } \\
\text { Análisis }\end{array}$ & Frecuencia & Porcentaje \\
\hline Si & 20 & 44,4 \\
No & 25 & 55,6 \\
Total & 45 & 100,0 \\
\hline \hline
\end{tabular}

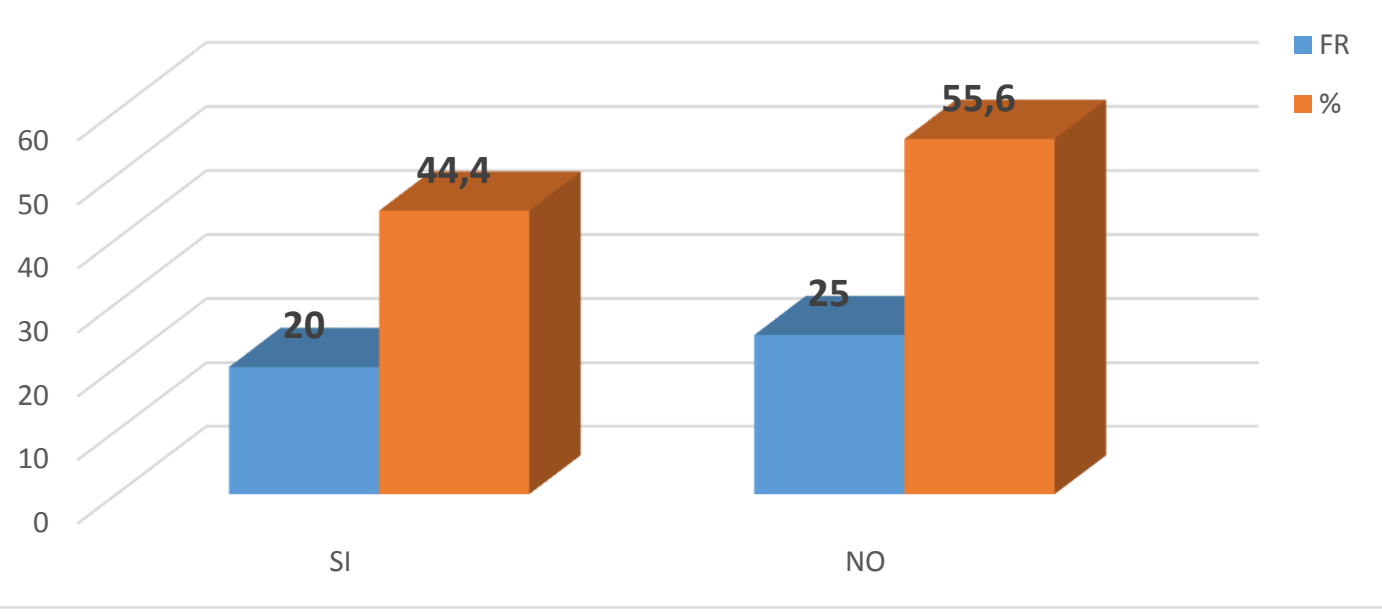

Grafico 3. Intervención de enfermería en la alimentación de pacientes diabéticos tipo II.

En la tabla y grafico 3, se presenta el análisis frecuencial del indicador intervención de enfermería en la alimentación de pacientes diabéticos tipo II que asistieron al centro de salud Daniel Acosta. En la misma se indica que el $55,6 \%$ no reciben recomendaciones acerca de la dieta que deben seguir en casa. $Y$ en menor porcentaje, con solo el $44,4 \%$ considero que no lo recibían. 
Tabla 4. Intervención de enfermería en la actividad física de pacientes diabéticos tipo II.

\begin{tabular}{lcc}
\hline $\begin{array}{c}\text { Alternativa/ } \\
\text { Análisis }\end{array}$ & Frecuencia & Porcentaje \\
\hline Si & 10 & 22,2 \\
No & 35 & 77,8 \\
Total & 45 & 100,0 \\
\hline \hline
\end{tabular}

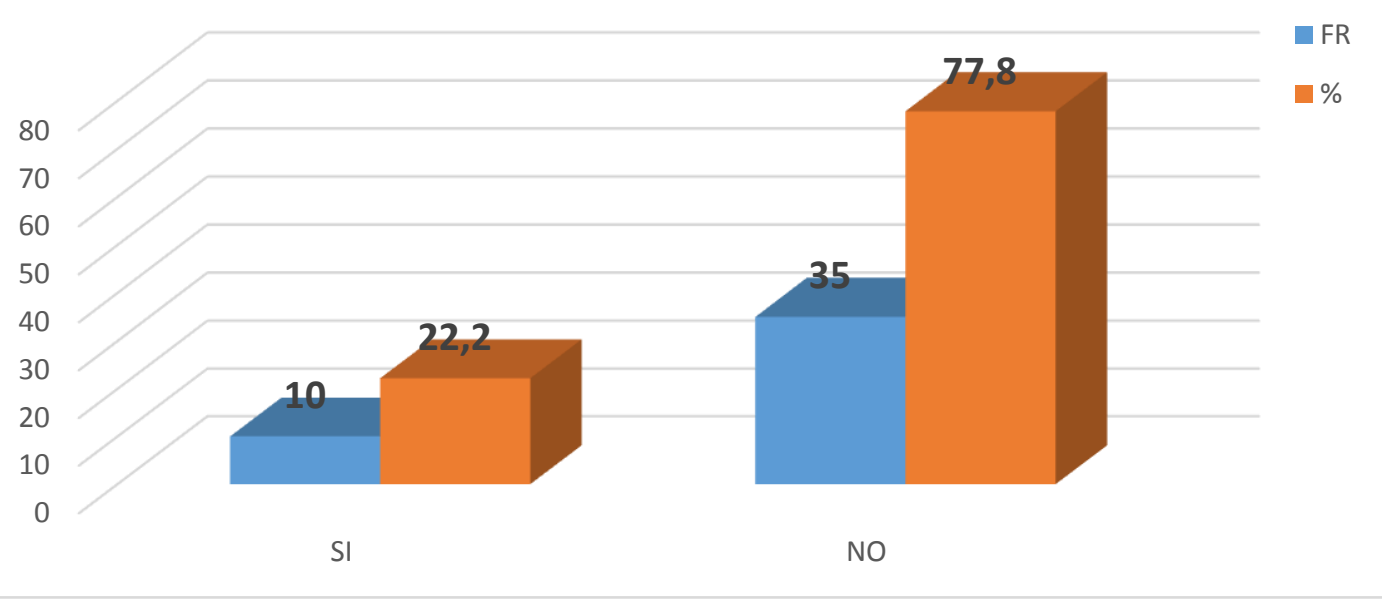

Grafico 4. Intervención de enfermería en la actividad física de pacientes diabéticos tipo II.

En la tabla y grafico 4, se presenta el análisis de la información sobre actividad física que se les brinda a estos pacientes, en este aspecto el $77,8 \%$ declaran que no se les educa en relación a este factor, mientras que solo el $22,2 \%$ expreso de forma positiva recibir información en este rubro. 
Tabla 5. Intervención de enfermería en educación sanitaria a la familia de pacientes diabéticos tipo II.

\begin{tabular}{lcc}
\hline $\begin{array}{c}\text { Alternativa/ } \\
\text { Análisis }\end{array}$ & Frecuencia & Porcentaje \\
\hline Si & 22 & 48,9 \\
No & 23 & 51,1 \\
Total & 45 & 100,0 \\
\hline \hline
\end{tabular}

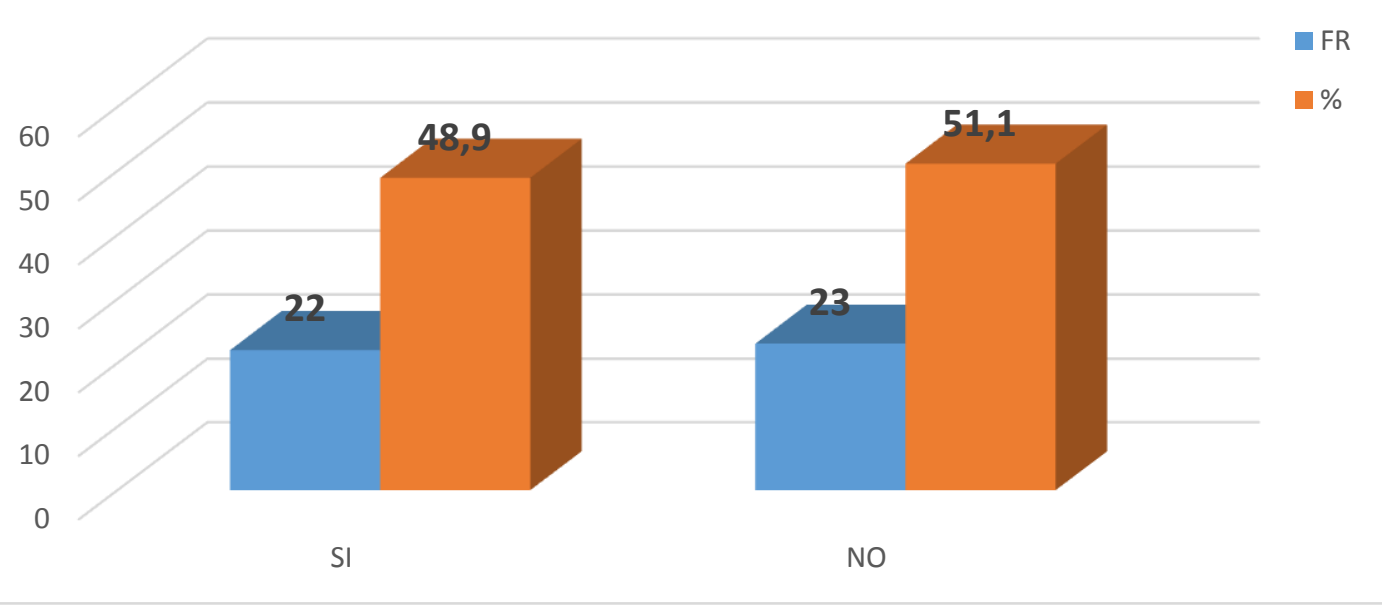

Grafico 5. Intervención de enfermería en educación sanitaria a la familia de pacientes diabéticos tipo II.

En la tabla y grafico 5 , se presentan los resultados del análisis frecuencial del indicador educación sanitaria a la familia, en la misma se indica que el $51,1 \%$ de los pacientes investigados coincidieron en opinar que no recibían educación sanitaria a seguir por la familia y en un porcentaje muy cercano del $48,9 \%$ indico de forma positiva. 


\section{CONCLUSIONES}

En atención a los resultados expuestos se pudo evidenciar lo siguiente:

Entre los pacientes investigados, en su mayoría de la tercera edad se constataron entre estos, dificultades en aspectos relacionados al apoyo social, bienestar personal, actividad física, soporte educativo, consumo de alimentos y afrontamiento de situaciones problemáticas.

La educación aplicada a una enfermedad crónica como la diabetes, facilita a la persona diabética y a su familia el aprendizaje de habilidades y conocimientos necesarios para asumir una actitud positiva frente a su enfermedad, potencia elecciones responsables para que asuman acciones de autocuidado, así como el apoyo necesario para que puedan formar parte activa del tratamiento.

La enfermería posee un rol determinante en el autocuidado de los pacientes diabéticos, al permitir prevenir, retrasar o disminuir la probabilidad de desarrollar complicaciones que generan tanto impacto en el ámbito social, psicológico y económico de las personas afectadas como a su entorno familiar.

\section{REFERENCIAS CONSULTADAS}

1. ADA (2005). American Diabetes Association. Standard Care in diabetes mellitus. Diabetes Care.2005;28(1).

2. ALAD (2011). Asociación latinoamericana de diabetes. Guías ALAD de diagnóstico, control y tratamiento de la diabetes mellitus tipo 2 con medicina basada en evidencia. Revista ALAD. Bogotá Colombia; 2011. Disponible en URL http://www.biblioteca-medica.com.ar/2011/02/guias-alad-dediagnosticocontrol-y.html

3. Baca B, Bastidas M De J, Aguilar M del R, De La Cruz M de J y González D M.(2008). Nivel de conocimiento del Diabético sobre su auto cuidado. Revista electrónica cuatrimestral de Enfermería Global. 13; 1-11.

4. Cabrera, E; Suarez, F y Díaz, H.( 2009). Propuesta para la clasificación fisiopatológica de la diabetes. Revista Cubana de Endocrinología. 2009(9)99109.

5. Casanova Moreno, M., Bayarre Vea, H. D., Navarro Despaigne, D. A., Giselda, S. R., Delgado, T., \& Maricela. (Julio-Septiembre de 2017). Efectividad de una estrategia educativa dirigida a adultos mayores diabéticos tipo 2 y proveedores de salud. Revista Cubana de Salud Pública, 43(3). 
Recuperado el 22 de ENERO de 2019, de Scielo salud publica: https://www.scielosp.org/article/rcsp/2017.v43n3/332-348/es/

6. Escobar, M (2000). Mitos sobre la prevención de las enfermedades no transmisibles en América latina. Revista de Salud Publica de México. Disponible en URL http:www.scielo.org/pdf/spw/v42nl/240/pdf

7. Galvis, RV.(2007). Guía De Atención De La Diabetes Tipo 2. Ministerio De Salud Dirección General De Promoción Y Prevención De La Salud. Colombia: MSDGPPS; 2007. p. 19-25.

8. García, A. (2002). Introducción a la metodología de la investigación. Editorial Trillas. México.

9. Girone, M (2005). Monitorización Clínica del Paciente Diabético. Actualización en medicina interna. ACMI 2005:14-19.

10. Hernández, M., Gutiérrez, J. P., y Reynoso, N. (2013). Diabetes mellitus en México: El estado de la epidemia. Salud pública México, 55(2), 129-136.

11. Hernandezi, R., Fernández, C., y Bautista, P. (2014). Metodología de la Investigación. México: Mcgraw-hill/Interamericana Editores. S.A. DE C.V.

12. Ministerio de Salud de Argentina. (2015). Recuperado el 12 de FEBRERO de 2019, de Guía de Práctica Clínica Nacional Diabetes Mellitus para el primer nivel de atención: http://www.msal.gob.ar/images/stories/bes/graficos/0000000076cnt-2012-0802 guia-breve\%20-prevencion-diagnostico-tratamiento-diabetes-mellitus-tipo2.pdf

13. OMS (2011). Organización Mundial de la Salud. Día mundial de la diabetes. USA [Internet] 2011 Disponible URL:http://www.worlddiabetesday.org./es

14. Ulloa Sabogal, I. M., Mejia Arciniegas, C. N., Plata Uribe, E. F., Noriega Ramírez, A., Quintero Gómez, D. L., y Grimaldos Mariñoll, M. A. (2017). Proceso de Enfermería en la persona con Diabetes Mellitus desde la perspectiva del autocuidado. Revista Cubana de Enfermería, 33(2). 